Article

\title{
Feasible Concept of an Air-Driven Fan with a Tip Turbine for a High-Bypass Propulsion System
}

\author{
Guoping Huang, Xin Xiang *, Chen Xia, Weiyu Lu and Lei Li \\ College of Energy and Power, Nanjing University of Aeronautics and Astronautics, \\ Nanjing 210016, Jiangsu, China; hgp@nuaa.edu.cn (G.H.); xiachen81@nuaa.edu.cn (C.X.); \\ lwy_651@nuaa.edu.cn (W.L.); lszyking@sina.com (L.L.) \\ * Correspondence: xin_chen89@sina.com; Tel.: +86-139-2144-7677
}

Received: 19 October 2018; Accepted: 27 November 2018; Published: 30 November 2018

check for updates

\begin{abstract}
The reduction in specific fuel consumption (SFC) is crucial for small/mid-size cost-controllable aircraft, which is very conducive to reducing cost and carbon dioxide emissions. To decrease the SFC, increasing the bypass ratio (BPR) is an important way. Conventional high-BPR engines have several limitations, especially the conflicting spool-speed requirements of a fan and a low-pressure turbine. This research proposes an air-driven fan with a tip turbine (ADFTT) as a potential device for a high-bypass propulsion system. Moreover, a possible application of this ADFTT is introduced. Thermodynamic analysis results show that an ADFTT can improve thrust from a prototype turbofan. As a demonstration, we selected a typical small-thrust turbofan as the prototype and applied the ADFTT concept to improve this model. Three-dimensional flow fields were numerically simulated through a Reynolds averaged Navier-Stokes (RANS)-based computational fluid dynamics (CFD) method. The performance of this ADFTT has the possibility of amplifying the BPR more than four times and increasing the thrust by approximately $84 \%$ in comparison with the prototype turbofan.
\end{abstract}

Keywords: high-bypass ratio engines; tip turbine; air-driven fan; aerodynamic analysis

\section{Introduction}

In recent years, small/mid-size cost-controllable aircrafts, such as unmanned aerial vehicles (UAVs), small airplanes for general aviation and cruising missiles, have demonstrated considerable value in the military/civilian fields [1,2]. The propulsion systems of these aircraft have two important requirements, namely, long voyage and low cost of use and manufacturing. As an important performance parameter, specific fuel consumption (SFC) is related to the two requirements. Several traditional techniques, such as increasing the overall pressure ratio (OPR), turbine inlet temperature and components efficiency, can be performed to improve the SFC. However, after an extensive development, these parameters have faced recent technology limitations, and raising the core thermal efficiency for conventional turbofan engines is becoming increasingly disadvantageous. In particular, increasing bypass ratio (BPR) is an appropriate method. Zimbrich in [3] suggests that, ideally, the SFC has a positive trend with increasing BPR, whereas the optimum fan pressure ratio has a continuous downward trend. Many segments of the aircraft industry and NASA (National Aeronautics and Space Administration) anticipate the application of high-bypass propulsion systems to small and fast transport aircraft $[4,5]$.

Therefore, a high-BPR turbofan engine will face the spool-speed conflict with a fan and a low-pressure turbine (LPT). For a given core size, a high BPR indicates an increasing fan diameter. On the one hand, considering the tip speed for an acceptable level of a fan's shock loss and the permissible centrifugal stress at a fan blade root, the rotational speed of a fan spool is limited at a low 
level. On the other hand, the LPT that drives the fan requires a high rotational speed to reduce stage loading and remain working at a high efficiency. This conflict will become increasingly aggressive with the increase in the BPR [6]. Several size problems will occur when the fan diameter is limited if the BPR is increased by scaling down the core engine size. For example, the blades of a high-pressure system (including the compressor and turbine) are too short, and the tip leakage losses consequently increase, thereby finally reducing the efficiency.

Several kinds of turbofan, such as a tri-spool turbofan and an aft-fan machine, have been developed for a high-BPR engine $[7,8]$. These kinds of turbofan can only relieve the conflict. However, when the BPR rises to ultra-high, the effects of the techniques in those engines are limited. The geared turbofan engine (GTF) is a successful technology for high/ultra-high BPR engines in commercial airplanes [9]. By using a gearbox, the GTF can enable the fan and LPT to rotate at different rotational speeds. The cost of developing the gearbox and improving the core spool is relatively small when compared with their benefits to commercial airplanes. In small/mid-size cost-controllable aircraft, applying the GTF may be another situation. The factors, including the limitation of a spool size, the cost of such a sophisticated geared-box, and the complicated rotor dynamics problems, will weaken the advantages of gearbox in the small/mid-size cost-controllable aircraft. In fact, the GTF has been used in several mid-size thrust engines, such as the TFE731 and ALF502, in the early 1970s. However, GTF is not extensively used at that time considering the cost and maintenance.

Tip turbine is another concept for solving the conflict and has been adopted in very high BPR turbofan engines. The mounting of the turbine at the tip of a fan blade can enable the fan and turbine to work at their optimal stage loading. In addition, the mechanical connection between the fan and the core rotor system is released through a gas-driven method, thus avoiding the changes in the core engine, especially the rotor spool. In the 1960s, the tip turbine has been used in lift fans for a vertical take-off and landing (VTOL) aircraft. NASA has investigated such a concept used in XV-5A and other further developed aircraft $[10,11]$. For the VTOL aircraft, the BPR of its engine must be high under a take-off condition to ensure the thrust against its weight. Reference [10] demonstrates that such a tip turbine cruise and lift fans can reach a BPR range of 10-100. NASA has conducted considerable research on such a kind of concept and has tested its turbine efficiency [12,13]. In the 2000s, researchers at Cranfield University studied a tip turbine-driven propulsion fan concept which has a potential future high-BPR turbofans [14]. Hatta [15] proposed a development study of an ATREX (Air-Turbo-Ram Expander cycle) engine, an air turbo ramjet with an expander cycle, which is a turbojet engine at a subsonic to Mach 2 flight. The ATREX uses a tip turbine configuration to provide compactness and light weight of the turbomachinery. According to existing public literature, a tip turbine functions at a high temperature and contains dozens to hundreds of blades. However, the heat conduction from a tip turbine to its fan will make maintaining a high efficiency difficult for a fan. An excessive number of blades will also impose additional stringent requirements on the strength of the leaf roots, especially the turbine blade at the tip. The reduction in the blade number of the tip turbine and its air temperature while ensuring driving capability will cause advantages to its use.

The present research proposed an aerodynamic consideration of an air-driven fan with a tip turbine (ADFTT) as a potential solution for a high-bypass propulsion system. The tip turbine had the same number of blades with its fan and works with bypass air from a core engine. The aerodynamic and thermodynamic analyses of such an ADFTT are conducted to show its potential in increasing BPR. As a demonstration, a typical small-thrust turbofan was selected as the prototype and applied the ADFTT to improve this model. The performances of the ADFTT were numerically simulated through the RANS based CFD method. 


\section{Principle of the ADFTT and Its Application to the High-Bypass Propulsion System}

\subsection{Principle and Brief Structure of the ADFTT}

The ADFTT is an aerodynamic component with two parts (Figure 1), namely, the fan and the tip turbine mounted at the tip of the fan. The tip turbine can achieve a positive circumferential velocity at a low spool speed. The two parts are attached to each other in a structural and aerodynamic design: the total pressure at the outlet of the fan and the turbine must be equal (minimizing the mixing loss); the blade numbers of the fan and the turbine rotor must be the same (considering the blade strength) and, given the structure consistency and reliability, the turbine rotor must have the same staking and sweeping laws with the fan rotor.

The blade of the air-driven fan rotor can be joined through two methods. The first method uses a blade shroud (Figure 1, (1) that can divide the flow paths of the fan and the turbine, in order to avoid the interferences. The second method uses a blade shoulder (Figure 1, (2) to join the fan and turbine blades. The blade shoulder is lighter than the blade shroud but presents more difficulties for its design. For the fan, the number of the blade will be limited because the small number of blades can reduce the centrifugal force on a fan disk and can lead to a small wetted area and minimal friction loss.

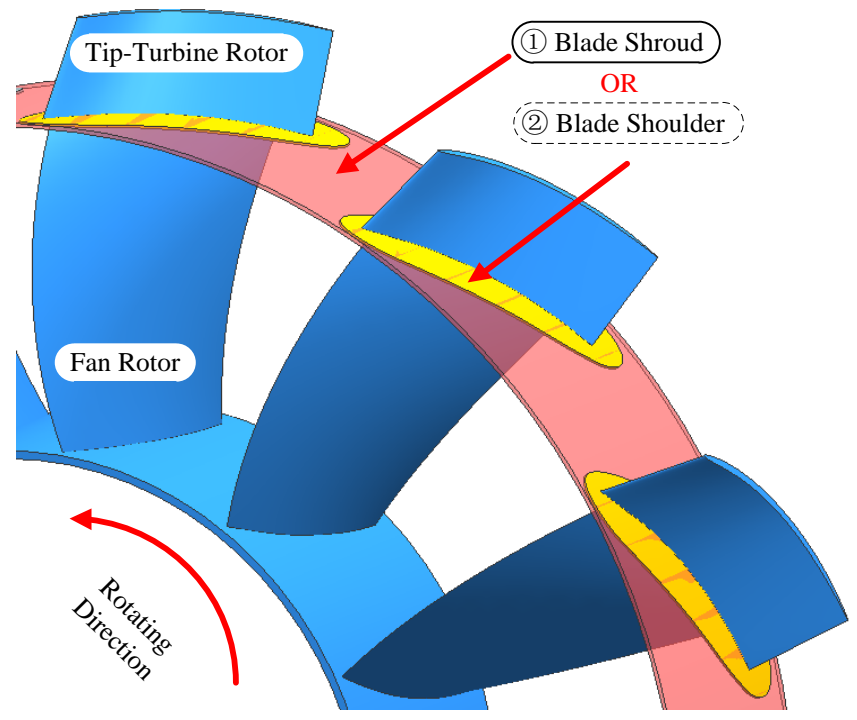

Figure 1. 3D Model of the ADFTT rotor.

\subsection{High-Bypass Propulsion System that Adopts the ADFTT}

The ADFTT can eliminate the mechanical limitations of a fan (which produces the optimum thrust) from the core engine and alter the energy transfer method aerodynamically. The air for driving the ADFTT can originate from the core engine, such as a turbojet, the bypass of turbofan and the mixed nozzle of the turbofan. Figure 2 illustrates a preliminary high-bypass propulsion system that adapts with the ADFTT. The system mainly includes five components, namely, (1) an air-driven fan rotor (including tip turbine), (2) an additional stator; (3) an air bleeding channel, (4) an additional casing and (5) a rotational casing. The air of the bypass of a core turbofan is transferred to the tip turbine (mounted at the tip of a fan blade) through the air-bleeding channel. Similar to the GTF which transfers the energy from the LPT to the airflow in the bypass, the ADFTT can also transfer energy from the bypass of a core engine to an increasing airflow in the additional bypass. The ADFTT rotates at a low speed and works as an 'aerodynamic geared box'. By using such an aerodynamic geared box, the mechanical limitations of the fan (which produces the optimum thrust) from the core engine can be removed, and the energy is transferred aerodynamically. Such a design concept is the subject of a Chinese invention patent [16]. 


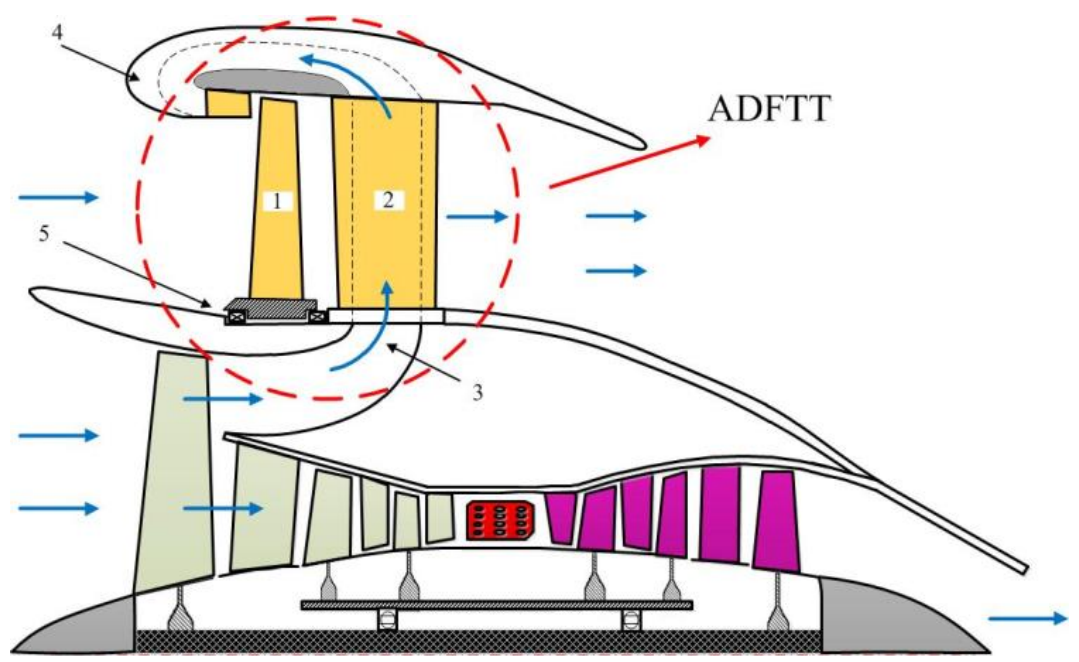

Figure 2. Structure sketch of the ADFTT applied to the prototype turbofan.

\section{Theoretical Thermodynamic Analysis of the ADFTT}

\subsection{Basic Equilibrium Principles}

As mentioned previously, the ADFTT acts as an aerodynamic box, whose basic function is to transfer energy to the airflow in additional bypass, and we defined its efficiency as $\eta_{A D F T T}$. To design this 'aerodynamic gearbox', the working procedures of the device should be analysed. Several basic equilibrium equations related to overall parameters can be listed as follows:

The energy transforming procedure is put forward at first place. The energy contained in the additional bypass originates from the bypass of the core engine, although the mass flow rate of the additional bypass is large. The energy equilibrium formula can be written as:

$$
E_{A D F T T}=\eta_{A D F T T} \cdot E_{B}=\eta_{A D F T T} \cdot m_{\text {bypass }} \cdot C_{p} \cdot T_{1}^{*}\left(\pi_{f 0}^{\frac{k-1}{k}}-1\right)=m_{A D F T T} \cdot C_{p} \cdot T_{1}^{*}\left(\pi_{A D F T T}^{\frac{k-1}{k}}-1\right),
$$

where $E_{A D F T T}$ is the energy that transformed by ADFTT (from tip turbine to air-driven fan), $m_{A D F T T}$ is the massflow rate of the ADFTT (including air-driven fan and tip turbine), $C_{p}$ is the specific heat capacity of air, $T_{1}^{*}$ is the total temperature of air of the inlet which equals to the ambient temperature, $\pi_{A D F T T}$ is the pressure ratio of ADFTT (after the mixing of air-driven fan and tip-turbine), $k$ is the specific heat ratio of air, $\eta_{A D F T T}$ is the equivalent isentropic efficiency of ADFTT, $E_{B}$ is the energy contains in the bypass flow of core engine, $m_{\text {bypass }}$ is the massflow rate of the bypass flow of core-engine, $\pi_{f 0}$ is the pressure ratio of the fan of core engine.

The relationship between $m_{A D F T T}$ and $m_{\text {bypass }}$ can be expressed as:

$$
B_{A D F T T}=\frac{m_{A D F T T}}{m_{\text {core }}}=\frac{B}{m_{\text {bypass }}} \cdot m_{A D F T T} .
$$

where the $B_{A D F T T}$ is the ratio of the massflow in additional bypass divided by the massflow of the high pressure system of core engine, $m_{\text {core }}$ is the massflow of the high pressure system of core engine, $B$ is the bypass ratio of core engine.

The power extracted from the tip turbine and the power consumed by the fan must be matching. The formula can be written as:

$$
m_{T T} C_{p} T_{f 0}^{*}\left(1-\frac{1}{\pi_{T T^{\frac{k-1}{k}}}}\right) \eta_{T T}=m_{A D F} C_{p} T_{1}^{*}\left(\pi_{A D F} \frac{k-1}{k}-1\right) / \eta_{A D F}
$$


where $m_{T T}$ is the massflow rate of tip turbine which is equals to the $m_{\text {bypass }}, \pi_{T T}$ is the pressure drop ratio of tip turbine, $\eta_{T T}$ is the efficiency of tip-turbine, $T_{1}^{*}$ is the total temperature of the inlet air of tip turbine, $m_{A D F}$ is the massflow rate of air-driven fan, $\eta_{A D F}$ is the isentropic efficiency of the air-driven fan.

These equations are the basic equilibrium equations of the ADFTT. Additional results can be deduced from these equations. For example, to minimise the mixing loss in the additional bypass nozzle, the total pressure of the fan and turbine outlets must be the same. Thus, the pressure ratios of the fan of core engine, the air-driven fan and tip turbine has a relationship as:

$$
\pi_{A D F}=\frac{\pi_{f 0}}{\pi_{T T}}=\pi_{A D F T T}
$$

With the help of these equations, we could calculate the overall parameters of ADFTT and get the performance of ADFTT.

\subsection{Thermodynamic Analysis of the ADFTT Effects}

A thermodynamic analysis is constantly the most important part that can show the loss and gain of a thermodynamic performance. An additional thermodynamic cycle to transfer energy will cost energy loss. We define the transfer efficiency for each turbofan to judge the amount of energy that the bypass airflow generates from the LPT. By ignoring the heat dissipation, we can easily deduce certain results from energy equations.

For a normal turbofan, the energy transfer efficiency is:

$$
\eta_{t}=\eta_{m} \eta_{f 0} \eta_{o}
$$

where $\eta_{m}$ is the mechanical efficiency of rotating shaft, $\eta_{0}$ is the efficiency of the outlet nozzle.

For the GTF, the energy transfer efficiency is:

$$
\eta_{G T F}=\eta_{m} \eta_{f 0} \eta_{o} \eta_{g}
$$

where $\eta_{g}$ is the mechanical efficiency of the geared box.

The ADFTT can be deduced as:

$$
\eta_{A D F T T}=\left(\frac{1}{\pi_{f 0}^{\frac{k-1}{k}}-1} \frac{\pi_{f 0}^{\frac{k-1}{k}}-\pi_{A D F}^{\frac{k-1}{k}}}{\pi_{f 0}^{\frac{k-1}{k}}} \eta_{T T} \eta_{f 0}+\frac{\pi_{f 0}^{\frac{k-1}{k}}-\pi_{A D F}^{\frac{k-1}{k}}}{\pi_{f 0}^{\frac{k-1}{k}}} \eta_{T T}+\frac{\pi_{A D F}^{\frac{k-1}{k}}-1}{\pi_{f 0}^{\frac{k-1}{k}}-1} \frac{\eta_{f 0}}{\eta_{A D F}}\right) \eta_{c h} \eta_{\text {mix }},
$$

where $\eta_{\text {mix }}$ refers to the equivalent efficiency due to the mix loss in the bypass, and $\eta_{c h}$ refers to the equivalent efficiency of the air-bleeding channel. This equation shows that the efficiency of the ADFTT is related to not only the components' efficiencies but also the pressure ratios (they could be deduced from other top-level parameters).

After the transfer procedure, the energy is redistributed into the airflow in the additional bypass with a large mass flow, and then thrust will be increased. The thrust of the prototype is:

$$
F_{\text {core }}=F_{\text {core-nozzle }}+F_{B}=(1+\alpha) F_{B}
$$

where $F_{\text {core }}$ is the thrust of core engine, $F_{\text {core-nozzle }}$ is the thrust produced by the nozzle of high pressure system of core engine, $F_{\mathrm{B}}$ is the thrust produced by the bypass of core engine (assume the core engine is separate exhaust), $\alpha$ refers to the ratio of the nozzle thrust divided by the bypass thrust. 
Considering the ideal situation and ignoring several small losses (e.g., the friction loss), the energy contained in the bypass is:

$$
E_{B}=\frac{m_{B} V_{B}^{2}}{2 \eta_{f 0}}=\frac{F_{B}^{2}}{2 m_{B} \eta_{f 0}},
$$

where $V_{B}$ is the airflow velocity of the outlet of bypass.

This part of the energy is redistributed by the ADFTT. Then, the thrust of the ADFTT can be expressed as:

$$
F_{A D F T T}=F_{\text {core-nozzle }}+F_{B}^{\prime}=\left[\alpha+\left(\frac{\eta_{A D F T T} \eta_{A D F} B_{A D F T T}}{\eta_{f 0} B}\right)^{1 / 2}\right] F_{B} .
$$

where $F_{B}^{\prime}$ is the thrust produced by additional bypass.

Thus, the thrust is amplified:

$$
\frac{F_{A D F T T}}{F_{\text {core }}}=\frac{\alpha+\left(\frac{\eta_{\text {ADFTT }} \eta_{A D F} B_{A D F T T}}{\eta_{f 0} B}\right)^{1 / 2}}{1+\alpha}=1+\frac{\left(\frac{\eta_{A D F T T} \eta_{A D F} B_{A D F T T}}{\eta_{f 0} B}\right)^{1 / 2}-1}{1+\alpha}
$$

Therefore, the ADFTT can increase the thrust by redistributing energy into an additional airflow, although the process consumes certain energy. In particular, the losses related to the fan, air-bleeding channel, tip turbine and air-driven fan are important. The thrust will increase with the BPR of the $\operatorname{ADFTT}\left(B_{A D F T T}\right)$ and will be influenced by an equivalent efficiency $\left(\eta_{A D F F F}\right)$. The $F_{A D F T T}$ calculated from Equation 11 is in ideal situation and ignored the losses in nozzle of additional bypass. Usually, the thrust will be decrease to approximately 0.98 due to the loss in nozzle. The effect of the increase in thrust under a typical condition is demonstrated in Section 3.3.

\subsection{Engine Performance Estimation of the ADFTT}

The efficiencies of the components are all important (as mentioned in Section 3.2), that is, the loss in the air-bleeding channel and the efficiencies of the tip turbine and air-driven fan. In this preliminary research, these efficiencies are assumed by the recent aerodynamic design level of components. For example, the efficiency of the air-bleeding channel is estimated at 0.96 because the bleeding channel with a positive pressure gradient can have a high pressure recovery under a low Mach number condition (e.g., less than 0.3). The efficiencies of the tip turbine and air-driven fan are conservatively estimated at 0.86 and 0.87 , respectively. Based on these efficiency estimations, $\eta_{A D F T T}$ is higher than 0.8. For example, a case has been studied on the basis of a prototype turbofan CF738 [17]. Several parameters at a design point are set to $\pi_{f 0}=1.7, \pi_{A D F}=1.09, \eta_{T T}=0.86, \eta_{f 0}=0.91, \eta_{A D F}=0.87$, $\eta_{c h}=0.96$ and $\eta_{A D F T T}=0.807$ in accordance with Equations mentioned above. Moreover, the BPR can be increased from 5.3 to 25 or higher (see it in Table 1). The efficiency of the 'aerodynamic geared box' seems unimpressive, the effect of amplifying the BPR is crucial and will reduce the SFC effectively. This result is similar with reference [18]: although the thermodynamic efficiency of geared fan is better than the tip turbine, the tip turbine arrangement remains a light and efficient system.

By applying the ADFTT concept, an exploratory design is studied on the basis of a prototype turbofan CFE738. Several top-level parameters are listed in Table 1. Several components' efficiencies at a design point are set as it is mentioned above. The design target is determined to ensure that the BPR is at approximately 27.0. The thrust is amplified by 1.89. As an exploratory design, the ADFTT remains its potential for further improvement on efficiencies of components (especially tip turbine and fan). Therefore, its ability to increase the bypass ratio is reflected, which is the main contribution to the thrust amplification. 
Table 1. Parameters of the prototype turbofan and ADFTT.

\begin{tabular}{ccc}
\hline Parameters & CFE738 & Preliminary ADFTT \\
\hline BPR & 5.3 & 27.8 \\
pressure ratio of bypass & 1.7 & 1.085 \\
Mass flow rate $(\mathrm{kg} / \mathrm{s})$ & 95.3 & 422.0 \\
Thrust $(\mathrm{kN})$ & 25.5 & 48.2 \\
$F_{A D F T T} / F_{\text {core }}$ & - & 1.89 \\
$\eta_{c h}$ & - & 0.96 \\
$\eta_{T T}$ & - & 0.86 \\
$\eta_{A D F}$ & - & 0.87 \\
$\eta_{A D F T T}$ & - & 0.807 \\
\hline
\end{tabular}

\section{Exploratory Design of the ADFTT}

\subsection{Top-Level Parameters of the Exploratory Design}

To demonstrate the ADFTT effects, an exploratory design is studied on the basis of the prototype turbofan of a general flight engine. The first step for exploratory design is to determine the top-level parameters of the propulsion system. These parameters determine the performance of the propulsion system and are used to guide the design of a component. Based on the theories in Section 3, we can estimate the performance of a turbofan and ADFTT approximately if several assumptions are introduced as follows:

(1) The core engine remains at the same performance (same thrust, same fuel consumption).

(2) All the energy transferred to the air of the (additional) bypass is converted into the kinetic energy of the air.

(3) Ignoring the nozzle loss to simplify the calculation.

From these top-level parameters, we can calculate the design parameters of each component, such as mass flow rate, pressure ratio/drop and efficiency. In fact, the equivalent efficiency of the ADFTT is crucial, and ensuring the efficiencies of the aerodynamic components to be as high as possible, especially for the tip turbine and fan, is necessary.

\subsection{Profiles Design of the ADFTT Blades}

The design processes of the fan and tip turbine, except the matching design process, are the same as the other turbomachinery; these design processes include preliminary, throughflow, 2D blading and 3D blading designs [19]. Notably, considering the structure consistency and reliability, the turbine rotor must have the same staking and sweeping laws with a fan rotor. The blade of the air-driven fan rotor can be joined through two methods. The first method uses a blade shroud (Figure 1, (1) which can divide the flow paths of the fan and turbine and avoid the interferences. The second method uses a blade shoulder (Figure 1, (2) which is lighter, but more challenging to design.

In accordance with the top-level parameters listed in Table 1, the aerodynamic parameters of the fan and turbine can be calculated predictively. Then, a geometry size and preliminary blade profile can be built. In this research, the ADFTT rotor, the most important and complex part, is completely built in the method (1). The first stator of the tip turbine is built to ensure the integrity of tip turbine.

Since the tip turbine mounted at the tip of the fan, the two parts are attached to each other in a structural and aerodynamic design. Several matching methods must be emphasised as follows: The blade numbers of fan and turbine rotor must be the same (considering the blade strength); the total pressure at the outlet of the fan and turbine must be equal (minimizing the mixing loss); the relative flow directions in each path should be similar (minimizing the shearing loss).

The profiles of the ADFTT are depicted in Figure 3 (they are the final profiles of the blades at present). In this exploratory design, we try not to restrict the design method of ADFTT or structure of blade, including the skewed and swept law. However, the fan has a skewed and swept blade to adapt the diameter changes from hub to tip. The rotor of the tip turbine is also a swept blade, whose swept 
law is consistent from the fan. On the other side, the stator of the tip turbine can be a straight blade, and only slightly skewed considering the diameter changes. All the blades are staking by the centre of gravity and the 3D structure of tip turbine and air-driven fan could be seen in Figure 1.

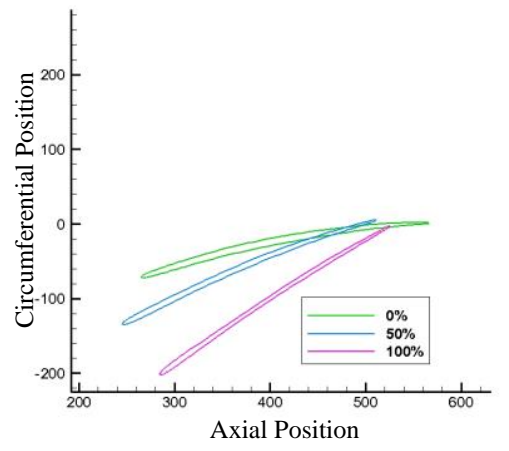

(a) Fan

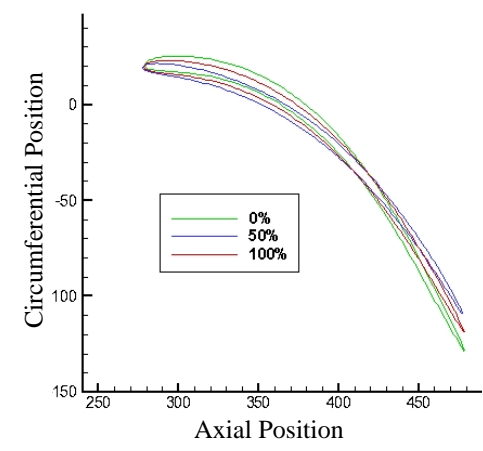

(b) Tip-Turbine Stator

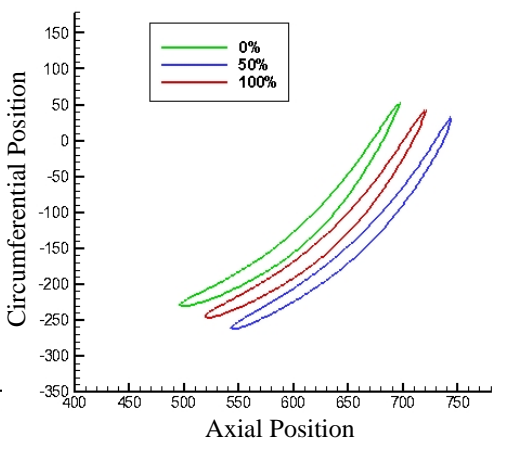

(c) Tip-Turbine Rotor

Figure 3. Section graphs of the ADFTT. (unit: $\mathrm{mm} ; 0 \%$ represents the hub; $100 \%$ indicates the tip).

For the fan, the number of blades will be limited so that the centrifugal force of the fan disk will be small. Also, a minimal number of blade leads to small wetted area and friction loss, which requiring to be considered when the fan pressure is low.

In accordance with the matching methods (1), the blade number of the tip turbine is limited by the fan. Then, the turbine blade can merge with the fan blade slightly and maintain the structure constraint which is positive for the blade strength. However, being mounted at the tip of the fan blade where the diameter is large, the solidity of the tip turbine is low. Preliminary calculation shows that the solidity of the tip turbine can be at approximately $0.6-0.7$ which is much less than a conventional turbine (>1.0) [20]. This low solidity problem may complicate the formation of a typical flow path for turbine blades. This topic will be discussed subsequently.

\section{Numerical Verification of the ADFTT Rotor and Discussions}

Currently, CFD plays an important role in the aerodynamic design of turbo machines. To evaluate the aerodynamic design of the ADFTT, an innovative and uncertain section, the ADFTT rotor (including the stator of the tip turbine), is numerically certificated through the CFD method. In addition, for an exploratory design, the air-bleeding channel is a new component and requires verification. However, considering that the channel is a positive pressure gradient, and the flow Mach number can be limited at a low level, we estimate that the difficulty of designing this channel is far less than the ADFTT rotor, and we will verify its design in a follow-up work.

\subsection{CFD Method}

Numeca, the commercial CFD software, including meshing module Autogrid and computing module Fine, is used for numerical simulation. An adiabatic and no-slip wall condition is adopted, and a periodic boundary is set for blade passage, thereby ignoring the clearance of a rotor blade.

The flow field was calculated using a 3D Reynolds Navier-Stokes equations method with a space-central difference scheme and four-step time advancement implemented using the Runge-Kutta method. There are several turbulence models such as Baldwin-Lomax model, Spalart-Almaras model, model and so on. Also, the turbulence can be fully resolved using direct numerical simulation (DNS) or partially resolved using large eddy simulation (LES). However, the computationally expensive of DNS and LES prevents them from being used as a design tool. The mesh resolution and quality requirements for this class of turbulence models are also less stringent than the ones required in DNS or LES. So those turbulence models are still wildly used. In this paper, the turbulence model is standard Spalart-Allmaras model, which is a one-equation model for the modified turbulent kinematic viscosity 
$\widetilde{v}$. As one-equation model, Spalart-Almaras model shows good agreement with airfoils [21] which are the basic of the blade of impellers and requires less computation resources than other two-equation models. The formulation of the model is the transport equation:

$$
\frac{\mathrm{D} \widetilde{v}}{\mathrm{D} t}=P-D+\frac{1}{\sigma}\left[\nabla \cdot\left((v+\widetilde{v}) \nabla \widetilde{v}+c_{b 2}(\nabla \widetilde{v})^{2}\right],\right.
$$

where $v=\mu / \rho$ is the laminar kinematic viscosity. The production and wall destruction terms read:

$$
\begin{gathered}
P=c_{b 1}\left(1-f_{t 2}\right) \widetilde{S} \widetilde{v} \\
D=\left(c_{w 1} f_{w}-\frac{c_{b 1}}{\kappa^{2}} f_{t 2}\right)\left[\frac{\widetilde{v}_{d}^{2}}{d} .\right.
\end{gathered}
$$

More details of such turbulent model could be obtained from the reference [21].

The Spalart-Allmaras model assumes that the mesh is sufficiently refined close to the wall surfaces with the non-dimensional wall distance $y+\sim 1$. However, in engineering application, it is difficult to insure all the mesh close to the wall surfaces with $y+\sim 1$. So the use of $y+$ within $1-10$ is a compromise and suggested in the Numeca manual. In our further work, the experiment study will be adopted, so the deviations between calculation performance (with this simulation setting) and actual performance could be known. Reference [22] checks the reliability of the calculation method and software, in which the fan has the similar rotating speed and size with the fan in this research.

The boundary conditions are set as follows: the total pressure of the inlet of tip turbine is $108,624 \mathrm{~Pa}$, the total temperature is $440 \mathrm{~K}$, and static pressure of its outlet is $101,300 \mathrm{~Pa}$; the total pressure of the inlet of air-driven fan is $101,325 \mathrm{~Pa}$, the total temperature is $293 \mathrm{~K}$, and static pressure of its outlet is 101,300 Pa; the adiabatic and non-sliding solid wall treatment method is adopted; the blade surface and hub surface are set as rotating boundary, the casing is static boundary, and the blade passage is set as periodic boundary.

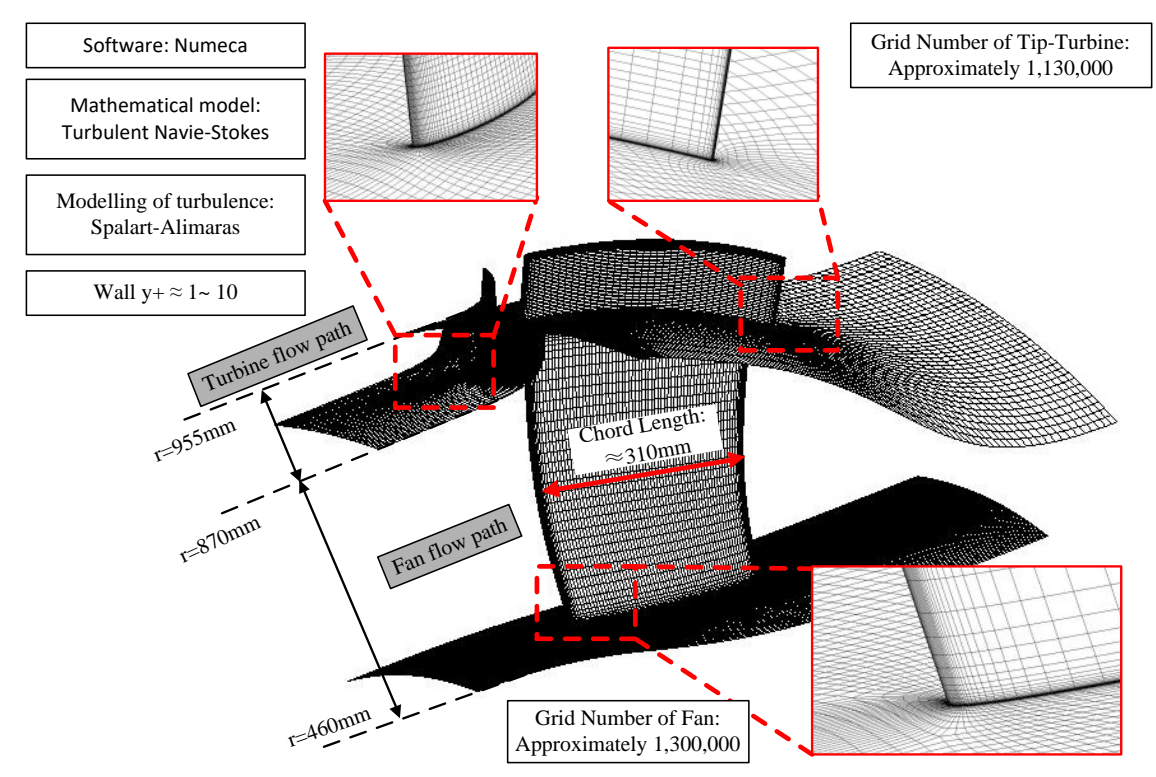

Figure 4. Medium Grid of the ADFTT Rotor (including the stator of the tip turbine).

To show the grid independence, three number grids (i.e., coarse, medium and fine) are constructed for the fan and tip turbine. The numbers of cells for the fan is 670,000 (coarse grid), 1,300,000 (medium grid) and 2,230,000 (fine grid) respectively. The numbers of cells for the tip turbine is 740,000 (coarse grid), 1,130,000 (medium grid) and 1,560,000 (fine grid) respectively. Figure 4 demonstrates the merged grid of the fan and tip turbine (medium grid). Figure 5 exhibits the results of grid number 
independence checking. The mass flow rates of both components remain the same with the increase in the number of grids, whereas the efficiencies of both components are nearly unchanged. The inlet Mach number and pressure ratio are also approximately the same with different grids for both components. The differences in the calculation results are nearly neglected. For the rapid preliminary design, less grid numbers will save plenty of time on the computation. However, a suitable number of grids will help reduce the deviation caused by the difference in the post processing of CFD results, so the medium grid is chosen for the present simulation.

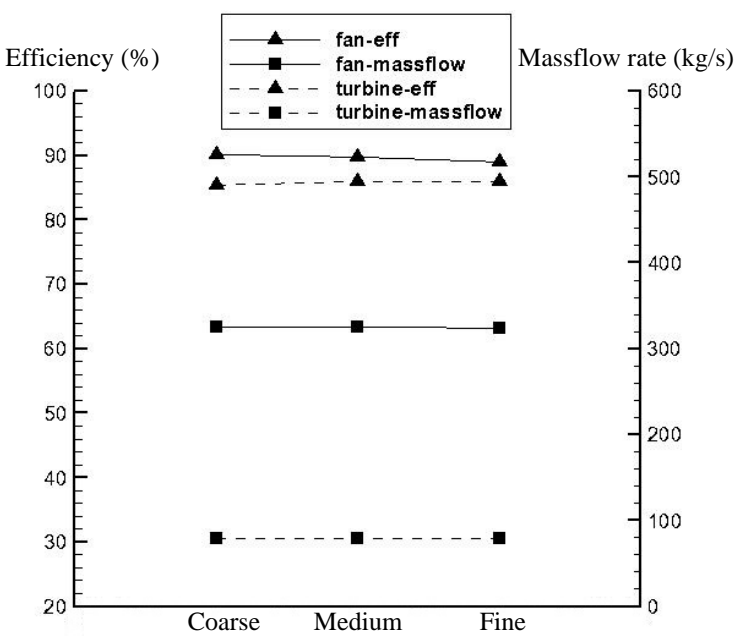

(a)

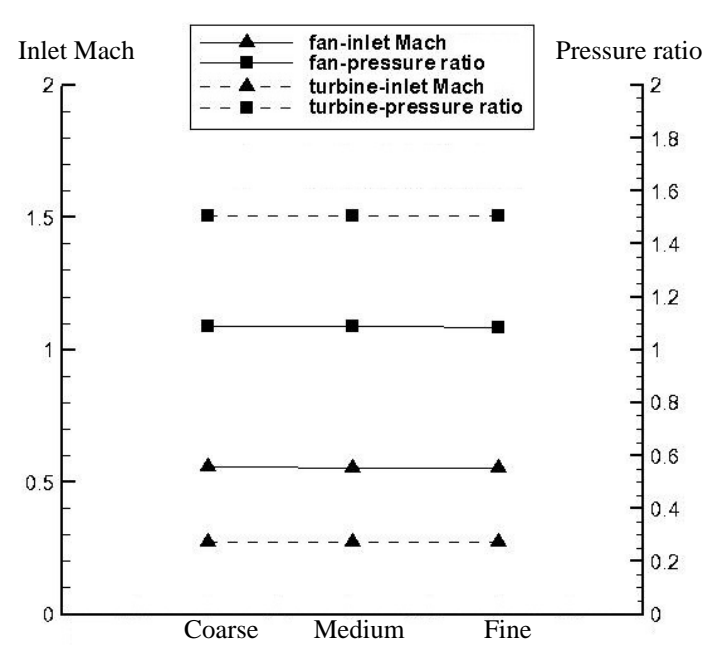

(b)

Figure 5. Grid independence results. (a) Efficiency and mass flow rate; (b) Inlet Mach number and pressure ratio.

\subsection{CFD Results and Discussions}

\subsubsection{Tip Turbine}

The blade number of stator is 29 and the blade number of rotor is 12 (equals to the fan rotor). Given the limitation in the blade number from an air-driven fan, the solidity of the tip turbine is too low (approximately 0.6-0.7) and cannot establish an integrated flow path by its adjacent blades if the turbine is designed through a normal design process of turbomachinery. Figure 6a displays the Mach number contour of the tip turbine which is designed through the normal process, and its isentropic efficiency is only approximately $70 \%$. The stator functions well and accelerates the flow to a high subsonic speed, but the rotor does not work well. The throat of the rotor flow path seems to be formed at Position A which is outside the flow path. The flow field shows a serious flow separation at the suction side of the blade and a large supersonic area at the downstream of the blade. Consequently, the conventional design method is an unsuitable solution for the tip turbine. The main reason is that blade solidity is too low to constrain airflow. In other words, the airflow deflects a large angle in this low solidity turbine and separation occurs in the latter part of the suction surface. 


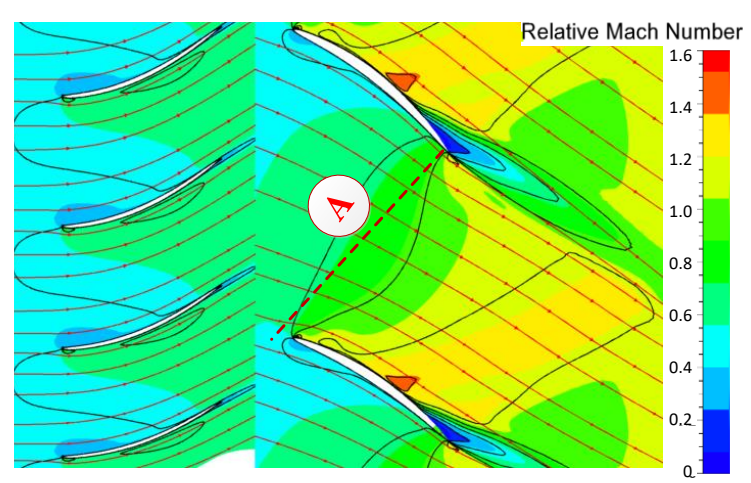

(a)

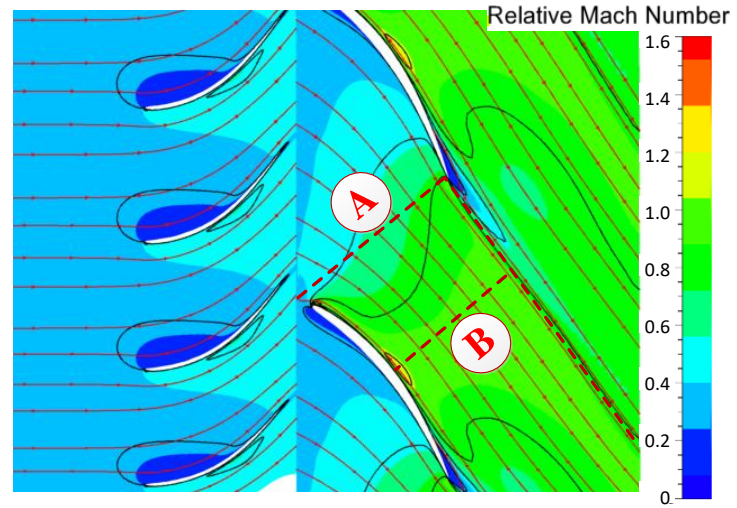

(b)

Figure 6. Mach Number Contour of the tip turbine (50\% span). (a) Conventional design; (b) High reaction design.

The reaction turbine is wildly used in gas turbine area for its positive pressure gradient in rotor [23]. High reaction turbine has two benefits: one is its positive pressure gradient; the other is the reducing the deflection angle of flow in the rotor. Both of the two benefits will make it difficult for airflow to separate. However, in high-reaction turbine, the relative velocity in rotor will be increased, which also limits the improvement of rotor's efficiency, even cause transonic or supersonic problems. So high-reaction turbine is seldom applied in practice.

To relieve the problem caused by such a low solidity of the turbine, a high reaction design method is adopted in tip turbine. The relative flow angle between the air and the axial direction in the rotor increases, and the turning angle of the air in the rotor can be controlled under a low level. The blade profile is improved through a direct problem method. The blade profile near the trailing edge must be optimised to ensure that the streamline at Position A is nearly parallel with the profile of a suction side at Position B. Figure $6 \mathrm{~b}$ presents the Mach number contour of the tip turbine that uses the aero-extending blade design. The physical throat seems to be located at Position A, but the flow field shows that the aero throat is located at Position B. Thus, the blade solidity is increased if the length of the blade is calculated until at least Position B with so called 'aero-extending method'.

The 'aero-extending' seems to be an apparent phenomenon of high-reaction design with trailing edge profile optimization. In addition, to reduce the impact of relative speed increase, the height of the turbine flow path is increased in the high reaction method (Figure 4 is the final grid of this high reaction design). The increasing of path height will brings two benefits, one is decreasing the flow velocity in stator and rotor (for the axial velocity is decreased), the other is reduce the flow velocity of the outlet of stator and let the air accelerate more in rotor. What's more, the tip turbine is not a high load or high pressure ratio drop turbine, so the relative velocity in rotor could be controlled at an acceptable level when high reaction turbine design is adopt. Though more losses will be produced due to the increasing of relative velocity of airflow, the improvement of turbine performance is still effective. The working conditions of the ADFTT make the low solidity tip turbine to be a special problem and could be fixed by this method.

Figure 7 shows more details of the flow field. The leading edge of hub is set as $0 \%$ section and the trailing edge of hub is set as $100 \%$ section. The $20 \%$ section is almost belongs to high pressure region (1), and most area of $100 \%$ section belongs to low pressure region (2). The high pressure region only covers the pressure side of blade, and the low pressure region only covers the suction side of the next blade. The air in the flow path of the rotor traverses two periods: Firstly, high pressure region, the flow is accelerated before Position A. Secondly, low pressure region, the flow is continue accelerated in the area between Positions A and B (the same with Figure 6b). In conventional turbine, the air usually flows through high pressure region (near suction side) or low pressure region (near suction side). However, in this tip turbine, the air flows through the high pressure region into the low pressure 
region. The flow field seems regular with only two small separations. One (S1) locates at the corner of tip near the leading edge. This separation is mainly caused by flow around leading edge (yellow stream lines). The other (S2) locates at the corner of hub near the trailing edge. This separation is mainly caused by the convolution of low velocity fluid of end wall. These two separations may be improved in the further, so that the efficiency of tip turbine could be further enhanced.

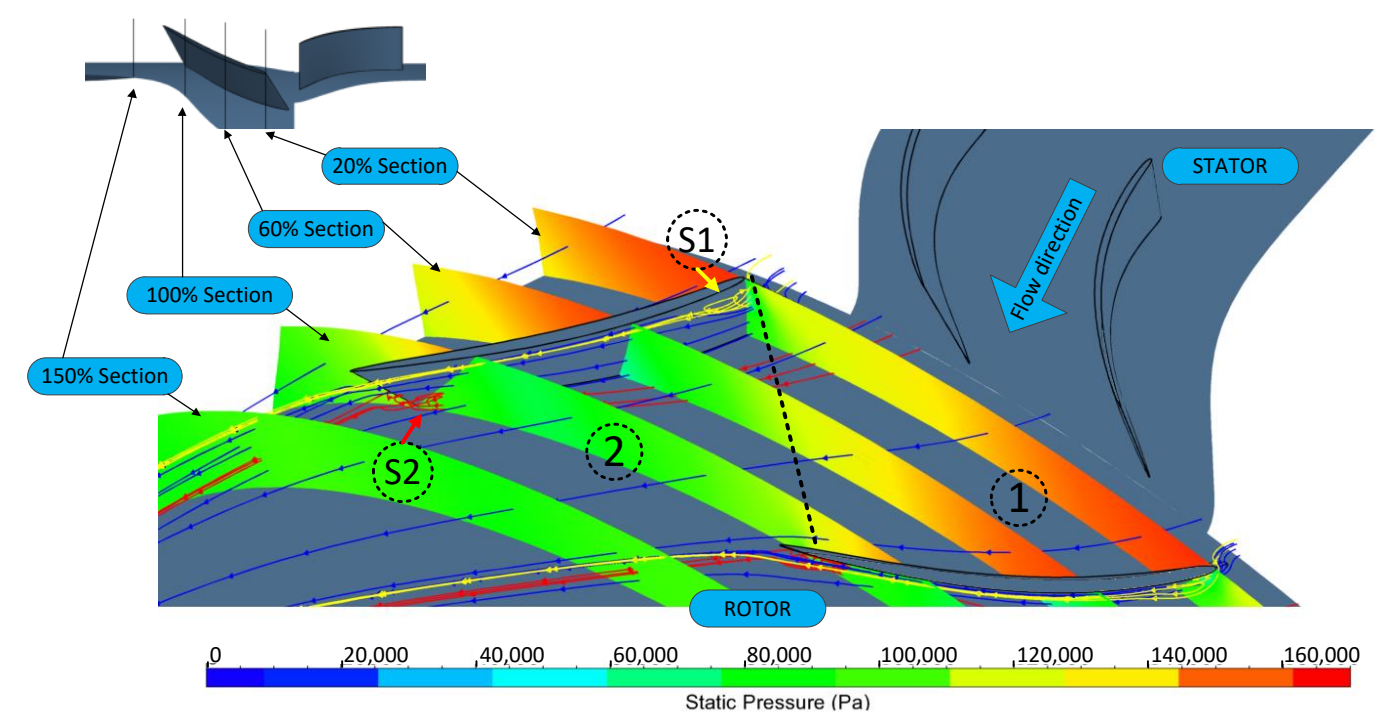

Figure 7. Static pressure contour of the cross stream of tip turbine at different sections.

Figure 8 shows the blade loadings of tip turbine at different spans. The blade loadings is almost the same with little differences at leading edge and position $B$. The pressure on suction side clearly shows the accelerate area from leading edge (near position $A$ ) to the middle of the blade (near position $B$ ). This phenomenal meets the flow field mentioned above. After position $B$, the air meets the adverse pressure gradient which could be one of the reasons for the corner separation at the trailing edge.

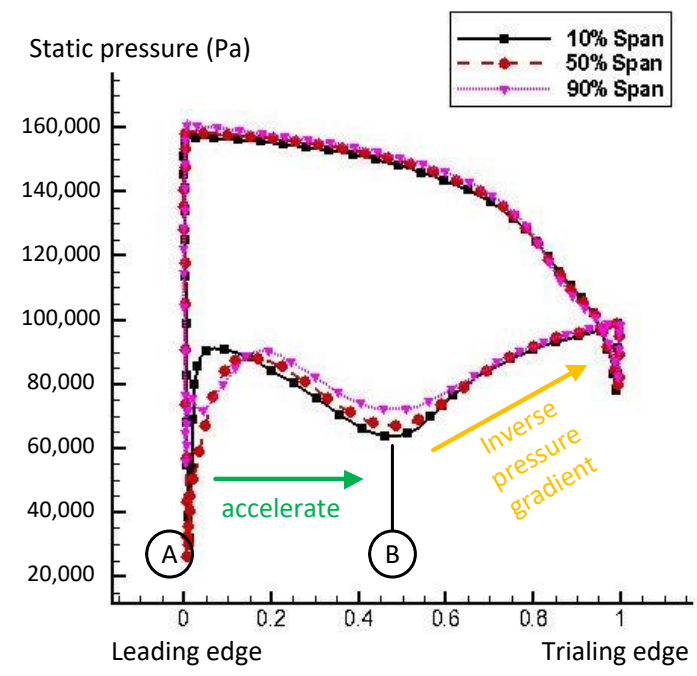

Figure 8. Blade loading of tip turbine at different spans ( $0 \%$ for hub and $100 \%$ for shroud).

Besides, as a result of the high reaction design, the absolute angle of the flow at outlet is $14.7^{\circ}$ (angle between the airflow direction and the axial direction, the same below). The normally designed turbine could be $0^{\circ}$. The flow direction angle must be turned in the additional stator to restore some thrust. Nevertheless, given the considerable efficiency improvement, the 'aero-extending' tip turbine 
blade remains impressive as a new method of turbine design. The performance of this tip turbine is summarised in Table 2 . The isentropic efficiency of this turbine is approximately $85.9 \%$ and satisfies the requirement of turbine performance. The design technique of the 'aero-extending' tip turbine blade will be investigated carefully in future research.

\subsubsection{Air-Driving Fan}

The blade number of fan rotor is 12 (equal to the turbine rotor). Since the pressure ratio of air-driven fan is low, more blades will lead to more friction loss and weight, but will not effectively improve the fan efficiency. Figure 9a illustrates the detailed flow field of the air-driven fan. There are two small separations (red and purple stream lines) near the end wells. Similarly, these separations are both caused by the convolution of low velocity fluid of end wall. There is a small supersonic region at the tip of the leading edge, which is caused by the flow around leading edge. Figure $9 \mathrm{~b}$ depicts the relative Mach number contour of the fan rotor at a $90 \%$ height span and shows the supersonic region. The relative Mach number of the entire flow field is nearly subsonic, and no flow separation or shock wave appears in the flow field. The main losses are friction and trailing wake. The relative flow direction is slightly turned by the fan rotor from $37.8^{\circ}$ to $26.3^{\circ}$, and the absolute flow angles changes from $0^{\circ}$ to $18^{\circ}$. This indicates that the circumferential velocity is minimal and the pressure ratio is low. In other circumstances, the air-driven fan rotor seems similar to a propeller because its solidity is much less than a conventional fan.

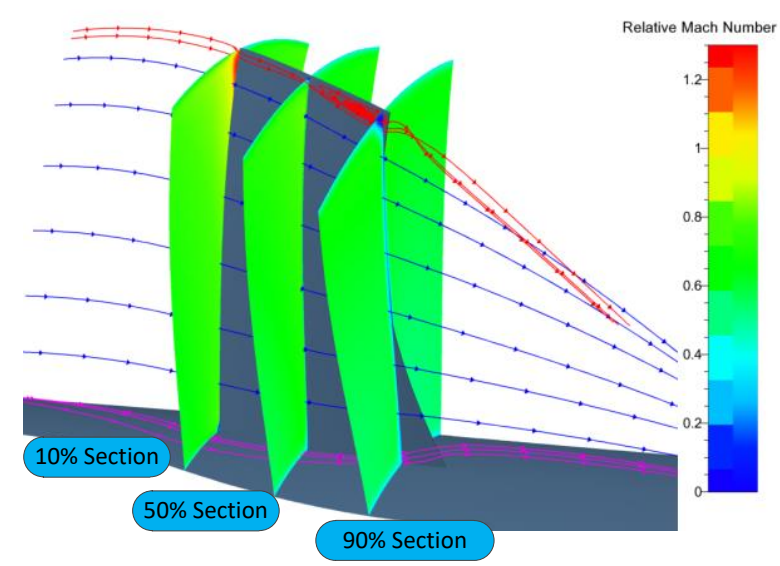

(a)

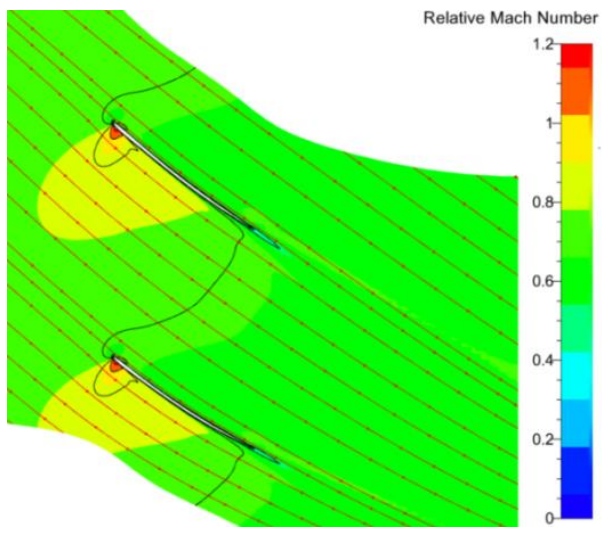

(b)

Figure 9. Flow Field Details of the Air-Driven Fan Rotor. (a) Mach number contour of the cross stream at different sections; (b) Mach number contour of at a $90 \%$ span.

Figure 10 shows the blade loading of air-driven fan at different span. The blade is a fore-loading blade. The loading at the front area reduces from hub to shroud, in order to reduce the acceleration of the flow near the tip. This is the benefit of the sweep blade. Since the circumferential velocity of the blade is increased with the increase of radius, faster acceleration at tip could lead to higher flow velocity and result in more losses, even shock wave. The profile of air-driven fan could continually be modified to get a more uniform loading and reduce the supersonic region near the leading edge.

Table 2. CFD Results of the air-driven fan and tip turbine.

\begin{tabular}{ccc}
\hline Parameters & Fan Rotor & Tip Turbine \\
\hline Efficiency & $89.7 \%$ & $85.9 \%$ \\
Pressure ratio (drop) & 1.09 & 1.51 \\
Mass flow rate $(\mathrm{kg} / \mathrm{s})$ & 325 & 79.2 \\
Power $(\mathrm{kW})$ & -2544 & 2654 \\
Torque $(\mathrm{N} \cdot \mathrm{m})$ & $-12,151$ & 12,672 \\
\hline
\end{tabular}




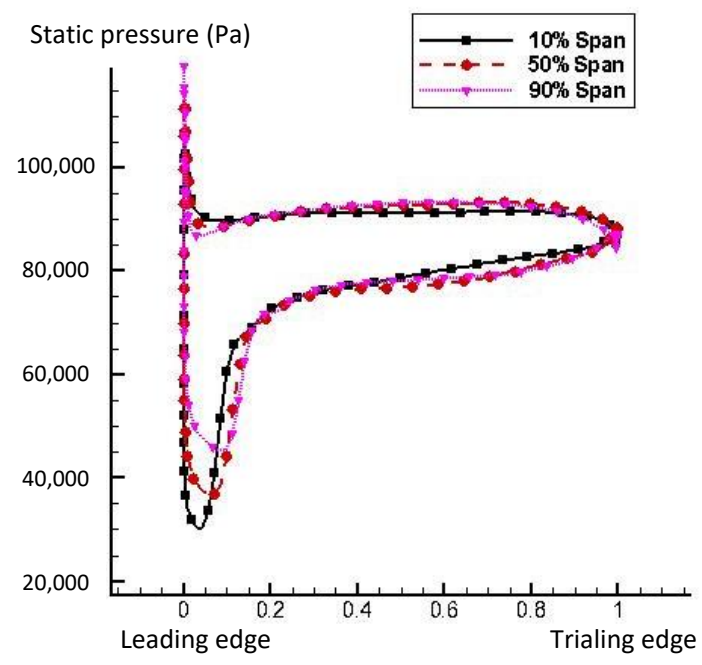

Figure 10. Blade Loading of Air-driven Fan at Different Span ( $0 \%$ for hub and $100 \%$ for shroud).

\subsection{Comparison between the Prototype Turbofan and the Demo ADFTT}

With the CFD results of the ADFTT, the prototype turbofan and the preliminary ADFTT can then be compared to indicate the performance advantages of the ADFTT. In Table 3, when the ADFTT is applied to the prototype turbofan, the BPR can be improved by $413 \%$, the thrust can be improved by $84.0 \%$ and the SFC can be reduced by $45.6 \%$. However, these results do not denote that the performance or the efficiency of the ADFTT is superior to the GTF or the conventional turbofan at the same BPR. In fact, in accordance with Equation (7), the equivalent efficiency of the ADFTT can be calculated. Therefore, the ADFTT, as the BPR 'amplifier', can truly significantly improve the performance of the prototype turbofan.

Table 3. Comparison between the Prototype Turbofan and the Preliminary ADFTT.

\begin{tabular}{cccc}
\hline Parameters & Prototype Turbofan & Preliminary ADFTT & Alteration (\%) \\
\hline BPR & 5.3 & 27.2 & +413.0 \\
Thrust $(\mathrm{kN})$ & 25.5 & 48.5 & +84.0 \\
\hline
\end{tabular}

After summarising the thermodynamic analysis, aerodynamic design and numerical simulation, the ADFTT is concluded as a feasible means for improving the performance of a turbofan. Further work must still be conducted to develop the detailed aerodynamic designs of components and multidisciplinary design methods of the ADFTT.

\section{Conclusions}

This research presents a conceptual design of an ADFTT for a high-bypass propulsion system. Through the work discussed above, the following conclusions can be drawn:

(1) A concept of a turbofan with an ADFTT is presented. This machine can solve the incompatibility problem of the fan and LTP, and is a promising device for high-bypass propulsion systems, especially for cost-controllable and small/mid-size aircraft.

(2) Theoretical thermodynamic analysis of the ADFTT is proposed. The equivalent efficiency is also introduced to evaluate the energy transfer process. The ADFTT significantly improves the bypass of the prototype turbofan and increase the thrust.

(3) An 'aero-extending' design can resolve the low solidity problem of the rotor of the tip turbine and ensure the positive performance. The aero-extending blade can extend the blade aerodynamically and achieve the design target of the efficiency. 
(4) This research aims to perform the exploratory design of the ADFTT, including the CFD results of the rotor of the ADFTT. The results of the demo work show the potential feasibility of the ADFTT, and further improvements can be expected. In comparison with the prototype turbofan, the performance of the CFD results shows that the ADFTT can improve the thrust by $84.0 \%$ and reduce the SFC by $45.6 \%$.

Author Contributions: Conceptualisation, G.H. and X.X.; methodology, X.X.; validation, X.X.; formal analysis, G.H.; investigation, X.X., W.L. and L.L.; resources, G.H.; data curation, X.X.; writing-original draft preparation, X.X.; writing-review and editing, G.H. and C.X.; visualisation, X.X. and G.H.; supervision, G.H.; project administration, G.H.; funding acquisition, G.H.

Funding: This research was funded by the National Natural Science Foundation of China (Grant number 51176072).

Acknowledgments: The authors express their gratitude to the Jiangsu Province Key Laboratory of Aerospace Power System (affiliated to the College of Energy and Power Engineering, Nanjing University of Aeronautics and Astronautics) for the technical support. The authors are also grateful to the team members of the College of Power and Energy of Nanjing University of Aeronautics and Astronautics for their cooperation.

Conflicts of Interest: The authors declare no conflict of interest.

\section{References}

1. Marin, N.; Spataru, P. The role and importance of UAV within the current theaters of operations. INCAS Bull. 2010, 2, 66-74.

2. Nicolosi, F.; Vecchia, P.D.; Corcione, S. Design and aerodynamic analysis of a twin-engine commuter aircraft. Aerosp. Sci. Technol. 2015, 40, 1-16. [CrossRef]

3. Zimbrich, R.A.; Colehour, J.L. An investigation of Very High Bypass Ratio Engines for Subsonic Transports. In Proceedings of the Propulsion Specialist Conference, Boston, MA, USA, 11-13 July 1988; Paper No. 88-2953.

4. Rohrbach, C.; Metzger, F.B.; Black, D.M.; Ladden, R.M. Evaluation of Wind Tunnel Performance Testing of an Advanced $45^{\circ}$ Swept Eight-Bladed Propeller at Mach Numbers from 0.45 to 0.85; NASA CR-3505; National Aeronautics and Space Administration: Washington, DC, USA, March 1982.

5. DeGeorge, C.; Turberg, J.; Wainauski, H. A Report on the Initial Testing of the Large Scale Advanced Prop-fan. In Proceedings of the 22nd Joint Propulsion Conference, Huntsville, AL, USA, 16-18 June 1986; AIAA Paper 86-1551. [CrossRef]

6. Larsson, L.; Grönstedt, T.; Kyprianidis, K.G. Conceptual Design and Mission Analysis for a Geared Turbofan and an Open Rotor Configuration. In Proceedings of the ASME 2011 Turbo Expo: Turbine Technical Conference and Exposition, Vancouver, BC, Canada, 6-10 June 2011; pp. 359-370.

7. Kestner, B.K.; Schutte, J.S.; Gladin, J.C.; Mavris, D.N. Ultra High Bypass Ratio Engine Sizing and Cycle Selection Study for a Subsonic Commercial Aircraft in the N + 2 Timeframe. In Proceedings of the ASME 2011 Turbo Expo: Turbine Technical Conference and Exposition, Vancouver, BC, Canada, 6-10 June 2011; pp. 127-137.

8. Neitzel, R. Comparisons of alternate energy efficient engines for future subsonic transports as affected by engine technology improvements. In Proceedings of the 12th Propulsion Conference, Palo Alto, CA, USA, 26-29 July 1976.

9. Kurzke, J. Fundamental Differences Between Conventional and Geared Turbofans. In Proceedings of the ASME Turbo Expo 2009: Power for Land, Sea, and Air, Orlando, FL, USA, 8-12 June 2009; pp. 145-153.

10. Kutney, J. Aerothermodynamic Considerations of the Tip Turbine Driving Lift/cruise Fan Propulsion System. In Proceedings of the AIAA Summer Meeting, Los Angeles, CA, USA, 17-20 June 1963.

11. Gleiter, D.P. A Comparison of Two Lift Cruise Fan Propulsion Concepts. In Proceedings of the AIAA Aircraft Systems and Technology Meeting, Dallas, TX, USA, 27-29 September 1976.

12. Haas, J.E.; Kofskey, M.G.; Hotz, G.M.; Futral, S.M., Jr. Cold-Air Performance of a Tip Turbine Designed to Drive a Lift Fan. I-Baseline Performance; NASA TM X-3452; National Aeronautics and Space Administration: Washington, DC, USA, 1976. 
13. Hass, J.E.; Kofskey, M.G.; Hotz, G.M. Cold-Air Performance of a Tip Turbine Designed to Drive a Lift Fan. IV-Effect of Reducing Rotor Tip Clearance; NASA TP-1126; National Aeronautics and Space Administration: Washington, DC, USA, 1976.

14. Casado, H.; Cristobal, E.; Lorido, A.; Ramsden, K.W. A Tip-Turbine Driven Propulsion Fan Concept. In Proceedings of the ASME Turbo Expo 2002: Power for Land, Sea, and Air, Amsterdam, The Netherlands, 3-6 June 2002; pp. 877-885.

15. Hatta, H.; Kogo, Y.; Tanatsugu, N.; Mizutani, T.; Ohnabe, H. Application of advanced carbon-carbon composites to a tip turbine structure of the ATREX engine. In Proceedings of the 36th Structures, Structural Dynamics and Materials Conference, Boston, MA, USA, 30 June 1997.

16. Huang, G.P.; Xiang, X.; Chen, J.; Chen, X.; Lu, W.Y. High Bypass Ratio Turbofan Engine with Air-Driven Fan. Chinese Patent NO:2014108237250, 8 October 2014.

17. Hu, X.Y. World Handbook of Small and Medium Aero Engines; Aviation Industry Press: Beijing, China, 2006.

18. Asmus, F.J. Design and development of the tip turbine lift fan. Ann. N. Y. Acad. Sci. 2010, 10, 147-176. [CrossRef]

19. Gallimore, S.J. Axial Flow Compressor Design. Proc. Inst. Mech. Eng. 1999, 213, 437-449. [CrossRef]

20. Glassman, A.J. Turbine Design and Application; NASA SP-290; NASA Special Publication: Washington, DC, USA, 1972; p. 290.

21. Spalart, P.; Allmaras, S. A one-equation turbulence model for aerodynamic flows. La Recherche Aerospatiale 1994, 1, 5-21.

22. Zhang, J.; Zhou, Z.; Wei, W.; Deng, Y. Aerodynamic design of an ultra-low rotating speed geared fan. Aerosp. Sci. Technol. 2017, 63, 73-81. [CrossRef]

23. Shepherd, D.G. Principles of Turbomachinery. Macmillan 1956. [CrossRef]

(C) 2018 by the authors. Licensee MDPI, Basel, Switzerland. This article is an open access article distributed under the terms and conditions of the Creative Commons Attribution (CC BY) license (http:/ / creativecommons.org/licenses/by/4.0/). 